\title{
Acupuncture for chronic stable angina pectoris based on the theory of Meridian- Viscera Association: study protocol for a multicenter randomized controlled trial
}

Zhaoxuan He ${ }^{1,2+}$, Yi Yang ${ }^{2,3+}$, Qiao Wen ${ }^{1,2+}$, Tao Yin ${ }^{1,2}$, Zhengjie Li $i^{1,2}$, Peihong Ma ${ }^{1,2}$, Hui Zheng ${ }^{1}$, Yunhong Yang ${ }^{1,2}$, Yongliang Jiang ${ }^{4}$, Jianqiao Fang ${ }^{4}$, Lei Lan ${ }^{1,2^{*}}$ and Fang Zeng ${ }^{1,2,5^{*}}$

\begin{abstract}
Background: Acupuncture has long been used to treat chronic stable angina pectoris (CSAP), but the acupuncture prescription for CSAP varies. This trial will compare the therapeutic effects between the acupoints on the Heart Meridian and the acupoints on the Lung Meridian for treating CSAP, so as to provide a better prescription for acupuncture treatment of CSAP.

Methods: This is a multicenter randomized controlled trial. A total of 148 CSAP patients will be randomly allocated into two groups through central randomization in a 1:1 ratio. This trial will include a 2-week screening period, a 4week treatment period, and a 4-week follow-up period. The primary outcome is the frequency of angina attacks from baseline to 4 weeks after inclusion. Secondary outcomes include the frequency of angina attacks from baseline to 4 weeks after acupuncture treatment, the pain intensity of angina, total ischemia burden, heart rate variability, QT dispersion, the score of Seattle Angina Questionnaire, and the score of Short-Form of McGill Pain Questionnaire. These outcome measures will be evaluated at baseline, at the end of acupuncture treatment, and at the end of follow-up.
\end{abstract}

Discussion: We hypothesize that the effectiveness of puncturing at acupoints on the Heart Meridian will not be the same as those on the Lung Meridian. The results will provide further evidence of Meridian-Viscera Association theory and references for acupoints selection in the clinical practice.

Trial registration: Chinese Clinical Trial Registry ChiCTR1900025804. Registered on September 9, 2019

Keywords: Chronic stable angina pectoris, Acupoint, Randomized controlled trial, Intradermal needle, MeridianViscera Association

\footnotetext{
* Correspondence: lanlei@cdutcm.edu.cn; zengfang@cdutcm.edu.cn

'Zhaoxuan He, Yi Yang, and Qiao Wen are co-first authors.

'Acupuncture and Tuina School/The 3rd Teaching Hospital, Chengdu

University of Traditional Chinese Medicine, 37\# Shierqiao Road, Chengdu

610075, Sichuan, China

Full list of author information is available at the end of the article
}

(C) The Author(s). 2020 Open Access This article is licensed under a Creative Commons Attribution 4.0 International License, which permits use, sharing, adaptation, distribution and reproduction in any medium or format, as long as you give appropriate credit to the original author(s) and the source, provide a link to the Creative Commons licence, and indicate if changes were made. The images or other third party material in this article are included in the article's Creative Commons licence, unless indicated otherwise in a credit line to the material. If material is not included in the article's Creative Commons licence and your intended use is not permitted by statutory regulation or exceeds the permitted use, you will need to obtain permission directly from the copyright holder. To view a copy of this licence, visit http://creativecommons.org/licenses/by/4.0/ The Creative Commons Public Domain Dedication waiver (http://creativecommons.org/publicdomain/zero/1.0/) applies to the data made available in this article, unless otherwise stated in a credit line to the data. 


\section{Background}

Chronic stable angina pectoris (CSAP) is a common manifestation of coronary artery disease (CAD) and serves as the initial symptom in approximately half of all patients with CAD [1-4]. In America, an estimated 15.5 million adults have chronic CAD, and more than 7 million adults suffer from angina [5]. CSAP is associated with an increased risk of major cardiovascular events and sudden cardiac death [6], significantly impacts the patients' quality of life (Qol) [7], and increases the considerable healthcare expenditure [8]. Currently, the main management of CSAP is pharmacological treatment including $\beta$-blockers, angiotensin-converting enzyme inhibitors (ACEI), angiotensin II receptor blockers (ARB), and antiplatelet medications and statins [9]. However, the efficacy of medications is not satisfactory for the ignored side effects [2].

Acupuncture has been used for cardiovascular disorders including precordial pain and palpitation for thousands of years $[10,11]$. The therapeutic effects of acupuncture for treating CSAP are gradually being accepted in Western counties. Recently, some randomized controlled trials (RCT) had investigated the clinic value of acupuncture for treating CSAP. For example, acupuncture as an adjunctive treatment to antianginal therapy could significantly alleviate angina compared to the waiting list [12]; acupuncture increased cardiac work capacity significantly compared to sham acupuncture, which expressed as dPRP (difference in pressure-rateproduct between rest and maximum exercise) and maximal PRP during exercise [13]; and acupuncture was superior to isosorbide dinitrate and nifedipine in improving the symptoms of coronary angina pectoris [14]. Since these studies proved the efficacy of acupuncture for CSAP from different aspects, how to improve the effectiveness and accessibility of acupuncture for CSAP attracts increasing attention.

According to the traditional Chinese acupuncture theory, meridians and viscera are closely related in physiological function, mutually affected in pathological changes and treatment. The Heart Meridian connects with the heart directly, and the Lung Meridian indirectly associates with the heart. Both of these two meridians can be used for cardiovascular diseases in clinical practice $[15,16]$. Based on the theory of Meridian-Viscera Association, our previous study found that not only the acupoints on the Heart Meridian and Pericardium Meridian, but also the acupoints on the Lung Meridian could improve the symptoms and the Qol of CSAP patients. To further optimize the acupoint prescription and provide more convenience and simple acupuncture therapy for CSAP, we designed this RCT. In this study, acupuncture prescription is composed of the basic acupoints (local acupoints close to the heart) and additional acupoints (acupoints on the Heart Meridian or the Lung Meridian), which are far from the heart. The aim of this study focuses on providing the reference for acupoint and acupuncture modality selection in acupuncture treating CSAP, so as to promote the application of acupuncture for treating CSAP. The intradermal needle has been selected as the acupuncture intervention for its advantages including continuous stimulation, high convenience, high safety, and non-invasiveness compared with the filiform needle $[17,18]$.

\section{Methods and design \\ Study design}

This is a two-arm, double-center, randomized controlled trial. A total of 148 CSAP patients will be enrolled and randomly assigned to two groups through central randomization in a 1:1 ratio. This trial will include a 2week screening period, a 4-week treatment period, and a 4-week follow-up period. Outcome assessments will be performed at the baseline, the end of treatment, and the end of follow-up (see Fig. 1 for the details of the study schedule).

The reporting of this trial is conducted according to the Standard Protocol Items: Recommendations for Intervention Trials (SPIRIT) guidelines [19] (Fig. 2). The Consolidated Standards of Reporting Trials (CONSORT) and the Standards for Reporting Interventions in Clinical Trials of Acupuncture (STRICTA) have been used as frameworks of methodology for the design of this protocol [20].

\section{Participants}

The CSAP patients will be recruited from two clinical centers, including the Hospital of Chengdu University of Traditional Chinese Medicine (CDUTCM) and the First Affiliated Hospital of Zhejiang Chinese Medical University (ZCMU).

All CSAP patients will undergo a careful physical examination including electrocardiogram (ECG), echocardiographic tissue Doppler imaging (TDI), blood pressure test, blood sugar test, routine test (blood, urine, and stool), biochemical blood test (ALT, AST, BUN, Scr), myocardial zymogram test (LDH, $\alpha-\mathrm{HBDH}, \mathrm{CK}, \mathrm{CKMB})$, serum immunoglobulin $\mathrm{E}$ (IgE), myoglobin (Myo), and cardiac troponin l (cTnl) before inclusion.

\section{Recruitment strategy}

Participants will be recruited from the outpatient and inpatient departments of Cardiology in the Hospital of CDUTCM and the First Affiliated Hospital of ZCMU from October 2019 to August 2021.

The recruitment strategies include advertisements in the post, leaflets, the website of CDUTCM (http:// 


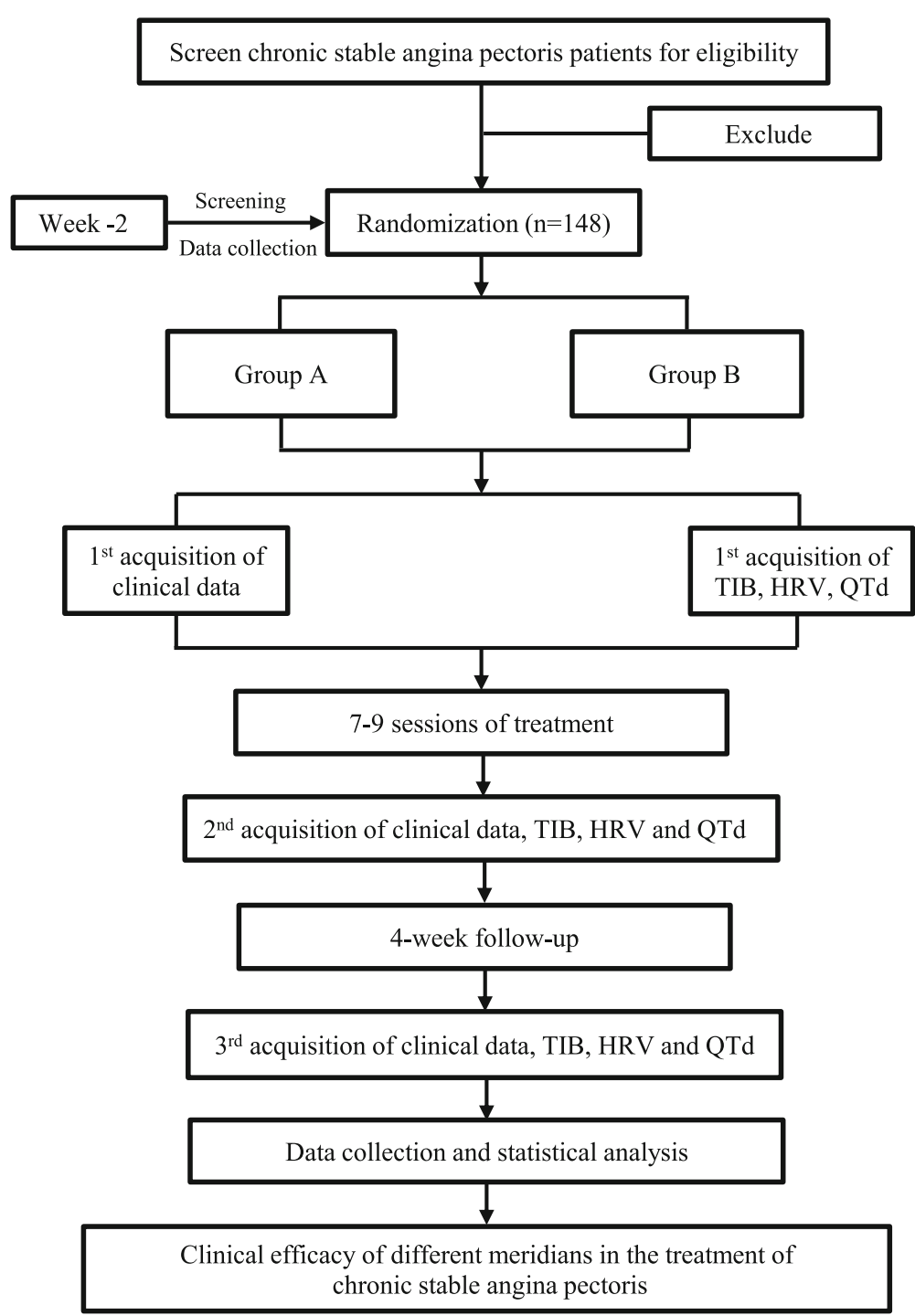

Fig. 1 Flowchart of the study design

cdutcm.edu.cn/), and ZCMU (https://www.zcmu.edu. $\mathrm{cn} /)$.

\section{Diagnostic criteria}

The diagnostic criterion bases on the Guideline for Diagnosis and Treatment of stable coronary artery disease (SCAD) issued by Chinese Society of Cardiology, Chinese Medical Doctor Association of Cardiovascular Physicians and Editorial Committee of Chinese Journal of Cardiology in 2018 [21].

\section{Inclusion criteria}

The patients will be included if they meet the following 6 items: (1) match the diagnostic criteria of SCAD; (2) meet the criteria of Class I or Class II according to the Canadian Cardiovascular Society (CCS) angina pectoris severity grade [22]; (3) age from 45 to 70 years; (4) have experienced angina attack in the last 3 months, and the attack frequency $\geq$ twice a week in the most recent month; (5) have a standardized medication history at least 2 months before enrolment (sustaining the same medication, usage, and dose), the basic therapeutic medication retains to one or more of the following: $\beta$-blockers ACEI, ARB, and antiplatelet medications and statins; and (6) have signed the informed consent.

\section{Exclusion criteria}

The patients will be excluded if they match the following 9 items: (1) be accompanied with acute myocardial 


\begin{tabular}{|c|c|c|c|c|c|c|c|}
\hline \multirow[b]{3}{*}{ TIMEPOINT } & \multicolumn{7}{|c|}{ STUDY PERIOD } \\
\hline & \multirow{2}{*}{$\begin{array}{c}\text { Screening } \\
\text {-2-week }\end{array}$} & \multirow{2}{*}{$\begin{array}{c}\text { Baseline } \\
\text { 0-week }\end{array}$} & \multicolumn{4}{|c|}{ Treatment } & \multirow{2}{*}{$\frac{\text { Follow-up }}{\text { 4-week }}$} \\
\hline & & & 1-week & 2-week & 3-week & 4-week & \\
\hline \multicolumn{8}{|l|}{ ENROLMENT: } \\
\hline Eligibility screen & $x$ & & & & & & \\
\hline Informed consent & $x$ & & & & & & \\
\hline Physical examination & $x$ & $x$ & & & & $x$ & $x$ \\
\hline Random allocation & & $x$ & & & & & \\
\hline \multicolumn{8}{|l|}{ INTERVENTION: } \\
\hline group A & & & $\longleftarrow$ & & & $\longrightarrow$ & \\
\hline group B & & & $\leftarrow$ & & & $\longrightarrow$ & \\
\hline \multicolumn{8}{|l|}{ ASSESSMENTS: } \\
\hline Angina diary & $x$ & $x$ & $x$ & $x$ & $x$ & $x$ & $x$ \\
\hline $\begin{array}{l}\text { The frequency of } \\
\text { angina attack }\end{array}$ & $x$ & $x$ & $x$ & $x$ & $x$ & $x$ & $x$ \\
\hline VAS & $x$ & $x$ & $x$ & $x$ & $x$ & $x$ & $x$ \\
\hline TIB & & $x$ & & & & $x$ & $x$ \\
\hline HRV & & $x$ & & & & $x$ & $x$ \\
\hline QTd & & $x$ & & & & $x$ & $x$ \\
\hline SAQ score & & $x$ & & & & $x$ & $x$ \\
\hline SF-MPQ score & & $x$ & & & & $x$ & $x$ \\
\hline SAS and SDS & & $x$ & & & & $x$ & $x$ \\
\hline $\begin{array}{l}\text { Expectation of } \\
\text { acupuncture }\end{array}$ & & $x$ & & & & & \\
\hline \multicolumn{8}{|l|}{$\begin{array}{l}\text { PARTICIPANTS } \\
\text { SAFETY: }\end{array}$} \\
\hline Adverse events & $x$ & $x$ & $x$ & $x$ & $x$ & $x$ & $x$ \\
\hline
\end{tabular}

Fig. 2 Standard Protocol Items: Recommendations for Interventional Trials (SPIRIT) schedule. VAS, visual analog scale; TIB, total ischemia burden; HRV, heart rate variability; QTd, QT dispersion; SAQ, score of Seattle Angina Questionnaire; SF-MPQ, Short-Form of McGill Pain Questionnaire; SAS, self-rating anxiety scale; SDS, self-rating depression scale

infarction, severe arrhythmia (advanced atrioventricular block, ventricular tachycardia, supraventricular tachycardia, frequent premature beats); (2) be accompanied with severe psychiatric illness; (3) fail to control hypertension (systolic blood pressure $\geq 140 \mathrm{mmHg}$ and/or diastolic blood pressure $\geq 90 \mathrm{mmHg}$ ) or diabetes mellitus (HbA1 above $7 \%$ in last 3 months); (4) have severe digestive, urinary, respiratory, hematological, nervous, or endocrine system diseases; (5) be pregnant or during lactation or intending to be pregnant in the 6 months; (6) have received acupuncture therapy due to cardiovascular illness in the last 1 month; (7) have the bleeding tendency and dermatitis; (8) be in the allergic constitution and be allergic to adhesive tape; or (9) be participating in any other clinical trials.

\section{Sample size}

In this study, we assume that $\mathrm{HO}$ represents the clinical effect of Group A is the same as Group B; H1 represents the effect of Group A is not the same as Group B.
According to the formula: $n=\frac{2 \sigma^{2}}{(\mu 1-\mu 2)^{2}} \times\left(\mu_{\alpha / 2}+\mu_{\beta}\right)^{2}$ [23]. In this study, assuming $\alpha=0.05$ (both sides), $1-\beta=$ 0.80 . Based on our previous study [12], $\mu 1$ is the improvement of the frequency of angina attacks in the acupoints on the disease-affected meridian group, and $\mu 2$ is the improvement of the frequency of angina attacks in the acupoints on the non-affected meridian group. With $\mu 1=5.57$ and $\mu 2=3.14, \sigma$ is estimated to be 5 , and the sample size is calculated as 67 per group. Considering a dropout rate of $10 \%$, we concluded that the sample size is 73.6 per group, and each group needs 74 cases after taking an integer. Therefore, a total of 148 CSAP patients will be finally recruited.

\section{Randomization}

To guarantee randomization concealment adequately, and not be influenced either by the acupuncturists or the participants, randomization will be performed by a clinical information management system (Beijing Bioknow Information 
Science \& Technology Co. Ltd., China). The investigators are trained to apply for randomization through text messages, online websites, or telephone application. When randomization information (including the participants' name, gender, and date of birth) is sent to the randomization center, the random numbers and group assignment will immediately be delivered to the acupuncturists.

\section{Blinding}

It is difficult to blind acupuncturists in acupuncture clinical trials. However, it is feasible to conceal the patients and group assignments from outcome assessors and statistical analysts. Patients are told that they will receive one of 2 effective acupuncture interventions after enrollment, and during the acupuncture treatment, patients in different groups will be in separate compartments to avoid communication. Outcome assessors and statisticians will be blind to the group allocations.

\section{Intervention}

All patients in the two groups will receive a total of 7-9 acupuncture sessions (at least 7 sessions) during 4 weeks. By intradermal needle, acupuncture stimulation will last for $48-72 \mathrm{~h}$ in each session with a 1 -day interval between each session.

The acupoint prescription will be composed of the basic acupoints and additional acupoints. The basic acupoints include bilateral Xinshu (BL15) and Juque (CV14), which are close to the heart. The basic acupoints will be used for the patients in both groups. The additional acupoints are all on the forearm, which are far from the heart relatively. For group A, 3 additional acupoints on the Heart Meridian will be selected, including bilateral Shenmen (HT7), Yinxi (HT6), and Tongli (HT5). For group B, 3 additional acupoints on the Lung Meridian will be selected, including bilateral Taiyuan (LU9), Lieque (LU7), and Kongzui (LU6) (Fig. 3).

The intradermal needle $(0.2 \mathrm{~mm}$ in diameter and 1.5 $\mathrm{mm}$ in length or $0.2 \mathrm{~mm}$ in diameter and $1.2 \mathrm{~mm}$ in length, Seirin Pyonex; Seirin Corporation, Shizuoka, Japan) will be inserted and retained in each acupoint for $48-72 \mathrm{~h}$ (at least retained for $48 \mathrm{~h}$ ). During the retaining period of the intradermal needle, patients will be requested to press the needle perpendicularly for 3 to 4 times every $24 \mathrm{~h}$, and each time lasts for around $30 \mathrm{~s}$. The manipulation will be performed according to the National Standard of the People's Republic of China "Standardized Manipulations of Acupuncture and Moxibustion-part 8: Intradermal needle" (GB/T 21709.82008) [24].

\section{Basic treatment}

The basic treatment for CSAP includes health education and primary medications, which will be performed from the baseline to the end of the follow-up period. Health education with CSAP aims to help the patients understand and master the basic knowledge of CSAP, such as risk factors, diagnosis, prevention, daily medication, and emergency treatment methods. Basic medications include $\beta$-blockers, ACEI, ARB, and antiplatelet medications and statins. Patients can choose one or several of the four medications as maintenance therapy according to cardiologists' advice.

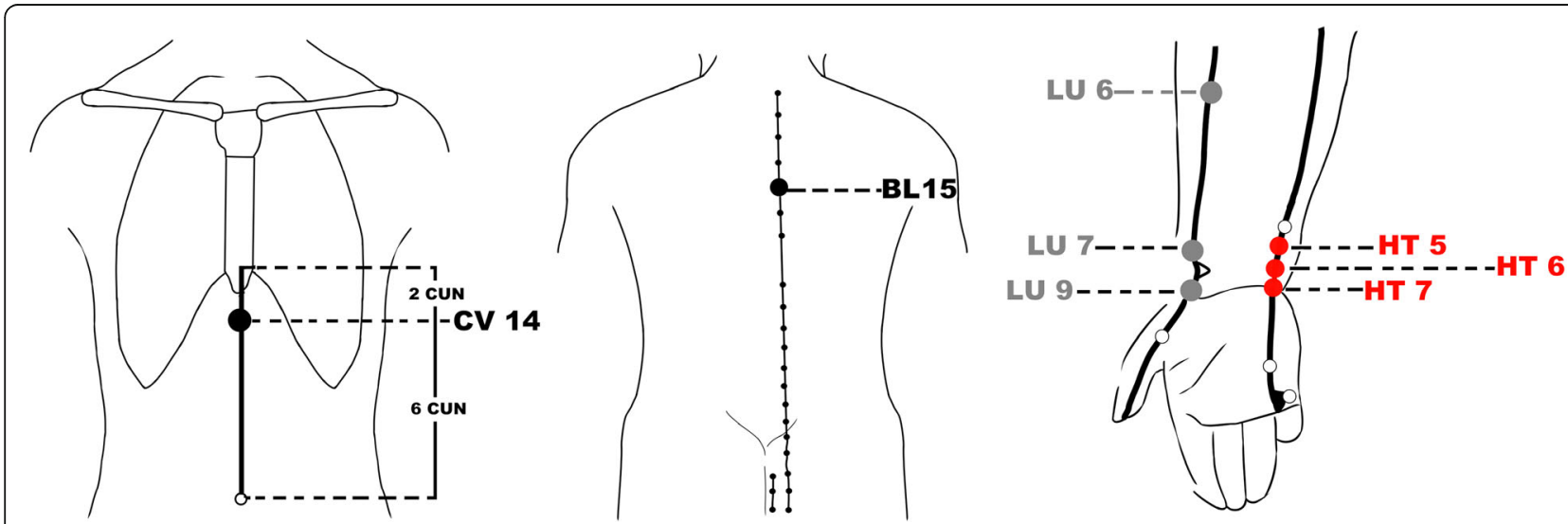

Fig. 3 Locations of Acupoints. CV14 (Juque), on the anterior meridian line of the upper abdomen,6 cun above the navel. BL15 (Xinshu), on the back,1.5 cun on the lateral to the lower border of the spinous process of the 5th thoracic vertebra. HT7 (Shenmen), on the wrist, at the ulnar end of the transverse crease of the wrist, in the depression on the radial side of the tendon of the flexor carpi ulnaris. HT6 (Yinxi), on the palmar side of the forearm, the point is on the radial side of the tendon of the flexor carpi ulnaris, 0.5 cun above the transverse crease of the wrist. HT5 (Tongli), on the palmar side of the forearm, the point is on the radial side of the tendon of the flexor carpi ulnaris, 1 cun above the transverse crease of the wrist. LU9 (Taiyuan), on the radial side of the transverse crease of the wrist, where the radial artery pulsates. LU7 (Lieque), on the radial margin of the forearm, 1.5 cun above the transverse crease of the wrist, between the brachioradial muscle tendon and the long abductor muscle tendon of thumb. LU6 (Kongzui), on the radial palmar side of the forearm, on the line connecting Chize (LU5) with Taiyuan (LU9),7 cun above the transverse crease of the wrist. 


\section{Outcomes}

\section{Primary outcome}

In this trial, the primary outcome is the frequency of angina attacks from baseline to 4 weeks after inclusion. The frequency of angina attacks will be recorded in the angina diary. The angina diary will be requested to complete and return to assessors in time by paper diary, emails, short messages, or telephone calls throughout the whole 10 weeks observation period.

\section{Secondary outcomes}

The secondary outcomes include: (1) the frequency of angina attacks from baseline to 4 weeks after the acupuncture treatment, (2) the pain intensity of angina which is assessed by visual analog scale (VAS), (3) total ischemia burden (TIB) and heart rate variability (HRV) which are recorded by 24-h dynamic electrocardiogram (DCG), (4) QT dispersion (QTd) which is recorded by ECG, (5) the score of Seattle Angina Questionnaire (SAQ), (6) the score of Short-Form of McGill Pain Questionnaire (SF-MPQ), (7) the score of self-rating anxiety scale (SAS), (8) the score of self-rating depression scale (SDS), and (9) the expectation values of acupuncture treatment which is composed of 7 questions and designed by our team. The VAS score will be extracted from the angina diaries. The second to eighth secondary outcomes will be evaluated at baseline, at the end of acupuncture treatment and at the end of followup. Moreover, the expectation of acupuncture treatment in each patient will be evaluated at baseline.

\section{Patient safety}

To guarantee the safety of patients, acupuncture treatment will be performed in the cardiology department where cardiovascular specialists and first-line physicians can participate in emergency timely. All adverse events (AEs) such as pain, allergy, bleeding, hematoma, and fainting should be recorded in the case report form (CRF) carefully. Serious adverse events (SAE) such as death or life-threatening events should immediately be reported to the principal investigator.

\section{Practitioners training and quality control}

All researchers involved in this trial must attend training classes to ensure all practices at each center are generalized and standardized according to the standard operating procedures (SOP). All the acupuncture manipulation will be performed by two licensed acupuncturists with clinical experience over 3 years. The acupuncturists should pass the examination on the SOP of acupuncture intervention before participating in this trial. In addition to the monthly inspection carried out by the research team, a specialized project supervision team will be established by the Clinical Research Center for
Acupuncture and Moxibustion of Sichuan Province, and responsible for monitoring the study every 3 months.

\section{Data management}

Data will be managed jointly by the two research centers. All the data will be recorded with printed and electronic case report forms CRFs. Only outcome assessors have access to CRFs and will perform the double-data entry.

\section{Data analysis}

All analyses will be strictly conducted according to the intention-to-treat (ITT) principle. Continuous variables will be described with means and standard deviation (SD) or median, maximum, minimum, P25, and P75. Categorical variables will be described with numbers and percentages (\%).

For the frequency of angina attacks from baseline to 4 weeks after inclusion, if the distribution conforms to the approximately normal distribution, analysis of covariance (ANCOVA) will be used. Since this study is a multicenter clinical trial, the center effects will be included in the analysis model. Age, gender, duration of the disease, and study site will be included in the model as covariates. Considering clustering effects of the number of angina attacks per patient, a hierarchical Poisson regression will be conducted exploratively. Separate ANCOVA analyses will be performed for secondary outcomes. The incidence of AEs in both groups will be compared with a Poisson regression model in which the number of participants with AEs is an independent variable and the group is a dependent variable.

Missing data will be imputed by multiple imputation. A sensitivity analysis using a pattern-mixture model will be conducted to assess the robustness of the results.

For all statistical analyses, Statistical Analysis System version 9.4 software (SAS Institute Inc., Cary, North Carolina) will be used. A $P$ value of less than $0.05(\alpha$ value of 0.05 , two-sides) will be considered statistically significant.

\section{Discussion}

This is the first study to evaluate the efficacy of acupuncture with intradermal needle for treating CSAP at different acupoint prescriptions. The results might deepen our knowledge on the theory of MeridianViscera Association and provide reference for the selections of acupoint prescriptions and acupuncture modality so as to develop better practice for acupuncture treating CSAP.

\section{The theory of Meridian-Viscera Association}

As an important component of the acupuncture theoretic system, the Meridian-Viscera Association theory 
emphasizes the close relationship between meridians and viscera in physiological function, pathological changes, diagnosis, and treatment. In detail, viscera physiological functions and pathological changes will manifest in the corresponding meridians or the acupoints [25], and the viscera disorders can be treated with the involved meridian and acupoints [26]. That means gastric disorders can manifest on the Stomach Meridian and be treated with the acupoints on Stomach Meridian. Cardiovascular disease can reflect on the Heart/Pericardium Meridian and be treated with the acupoints on the Heart /Pericardium Meridian. A number of modern studies have confirmed the relatively specific relationship between meridians and viscera and the modulating effect of meridians/acupoints for the corresponding viscera functions [27, 28]. For example, our previous study found that puncturing at the acupoints on the Stomach Meridian can effectively improve the symptoms and QoL of the functional dyspepsia (FD) patients compared to puncturing at sham acupoints or the acupoints on the Gallbladder Meridian [29].

The CSAP pertains to heart-related diseases. Acupuncture treatment can be performed on the meridians/acupoints which is related to the heart. In light of the traditional Chinese acupuncture theory, the Heart Meridian is directly related to the heart, and the Lung Meridian is indirectly connected to the heart. The acupoints on these two meridians can all be used for cardiovascular diseases. Therefore, this study tries to compare the therapeutic effects of the acupoints on the Heart Meridian (direct-related meridian) and the acupoints on the Lung Meridian (indirect-related meridian) for treating CSAP.

\section{Acupoints selection}

In clinical practice, selecting the local points and distal points simultaneously is the basic principle for developing acupoint prescriptions. In this study, the local acupoints, including Xinshu (BL15, the Back-shu point of the heart) on the back and Juque (CV14, the Front-mu point of the heart) on the chest, will be selected as the basic points because they are close to the heart and are the most commonly used local acupoints for cardiovascular diseases. For the distal acupoints, 3 acupoints on the Heart Meridian or on the Lung Meridian will be selected respectively as the additional acupoints. They all locate on the forearm, which is far from the heart. They are the Shenmen (HT7), Yinxi (HT6), and Tongli (HT5) on the Heart Meridian for group A and the Taiyuan (LU9), Lieque (LU7), and Kongzui (LU6) on the Lung Meridian will be selected respectively for group B. According to the literature analysis $[16,28]$, these 6 acupoints were all used for treating cardiovascular diseases. Among them, Shenmen (HT7) and Taiyuan (LU9) are the Yuan-source points of the Heart Meridian or the Lung Meridian, respectively; Tongli (HT5) and Lieque (LU7) are Luo-connecting points of the Heart Meridian or the Lung Meridian, respectively; and Yinxi (HT6) and Kongzui (LU6) are Xi-cleft points of the Heart Meridian or the Lung Meridian, respectively.

\section{Acupuncture modality selection}

Intradermal needle is a kind of special acupuncture needle for superficial puncture, which could be inserted perpendicularly into the skin with a tiny needle and be fixed with a piece of adhesive tape for 1 to 3 days to produce a gentle continuous stimulation on acupoint [30]. Compared with the filiform needle, the intradermal needle has more convenient operation, less pain, and fewer adverse reactions $[17,18]$. So the intradermal needle has recently been widely used for chronic diseases such as chronic tension-type headaches and insomnia [31, 32]. In addition, a small sample size clinical study also indicated that the intradermal needle could significantly improve the clinical symptoms of CSAP [33]. Therefore, considering the simplicity of the operation and the continuity of stimulation, the intradermal needle has been selected as the acupuncture modality in this study.

\section{Basic treatments with medicines}

The guidelines from the Chinese Society of Cardiology and the American College of Cardiology/American Heart Association (ACC/AHA) hold that the basic treatment of CSAP mainly includes health education and primary drugs $[21,34]$. So in this study, health education and medications are selected as the basic treatment for the patients with CSAP.

In this study, all patients are recommended to modify their lifestyles and control unhealthy habits that might affect diseases, such as limiting alcohol consumption, controlling weight, and quitting smoking. Health education is beneficial for the patients to be aware of the disease, to correct unhealthy behaviors and eating habits in daily life, and to avoid various accidents or adverse conditions during treatment and improve the safety of treatments.

Based on the guidelines [21,34] and cardiologists' recommendations, this study will select the four most commonly used medications as basic medications for treating CSAP, including $\beta$-blockers, ACEI, ARB, and antiplatelet medications and statins.

In conclusion, focuses on the theory of MeridianViscera Association, this study tries to investigate the efficacy differences in improving the symptoms of CSAP between two commonly used meridians (acupoints prescription), so as to provide evidence for the selections of acupoints and acupuncture modality. The results might 
be valuable for developing good practice for acupuncture treating CSAP.

\section{Trial status}

This trial was registered on 9 September 2019 at the Chinese Clinical Trial Registry (Registration number: ChiCTR1900025804, the protocol version number: F2.0). The trial is currently in the stage of patient recruitment. The first patient was included on 11 October 2019. To date, 12 patients have been included. Affected by the outbreak of Corona Virus Disease 2019 (COVID-19), recruitment will be approximately completed before 30 August 2021 and the trial is estimated to finish in November 2021.

\begin{abstract}
Abbreviations
CSAP: Chronic stable angina pectoris; CAD: Coronary artery disease: Qol: Quality of life; ACEl: Angiotensin-converting enzyme inhibitors; ARB: Angiotensin II receptor blockers; RCT: Randomized controlled trial; dPRP: Difference in pressure-rate-product between rest and maximum exercise; SPIRIT: Standard Protocol Items Recommendations for Intervention Trials; CONSORT: The Consolidated Standards of Reporting Trials; CDUTCM: Chengdu University of Traditional Chinese Medicine; ZCMU: Zhejiang Chinese Medical University; ECG: Electrocardiogram; TDI: Echocardiographic tissue Doppler imaging; IgE: Serum immunoglobulin E; Myo: Myoglobin; CTnl: Cardiac troponin I; SCAD: Stable coronary artery disease; CCS: Canadian Cardiovascular Society; VAS: Visual analog scale; TIB: Total ischemia burden; HRV: Heart rate variability; DCG: Dynamic electrocardiogram; QTd: QT dispersion; SAQ: Score of Seattle Angina Questionnaire; SF-MPQ: Short-Form of McGill Pain Questionnaire; SAS: Selfrating anxiety scale; SDS: Self-rating depression scale; AEs: Adverse events; SAE: Serious adverse events; CRF: Case report form; SOP: Standard operating procedures; ITT: Intention-to-treat; SD: Standard deviation; ANCOVA: Analysis of covariance; SAS: Statistical Analysis System; FD: Functional dyspepsia; ACC/ AHA: The American College of Cardiology/American Heart Association; COVID-19: Coronavirus disease
\end{abstract}

\section{Acknowledgements}

We thank Dehua Li, Ying Lan, Rong Hu, Wen Xie, and the Hospital of Chengdu university of TCM, for the recommendations of diagnostic criteria, inclusion criteria, and primary drug selection.

\section{Authors' contributions}

Fang Zeng and Lei Lan are responsible for this study. Zhaoxuan He, Yi Yang, Qiao Wen, Zhengjie Li, and Tao Yin conceived and designed the study. Zhaoxuan He, Yi Yang, and Qiao Wen participated in drafting the trial protocol and preparing the manuscript. Peihong Ma, Hui Zheng, and Yunhong Yang planned a data analysis solution. Yongliang Jiang and Jianqiao Fang participated in the data collection, recruitment, and treatment of patients in the subcenter. The authors read and approved the final manuscript.

\section{Funding}

The trial is financially supported by the National Key Research and Development Program (NO.2018YFC1704600, NO.2018YFC1704605). The funders have no role in the trial in terms of the study design, data collection, management, analysis, interpretation, and manuscript writing.

\section{Availability of data and materials}

This trial does not involve the storage of biological specimens. The data and materials during the current study are available from the corresponding author on reasonable request.

\section{Ethics approval and consent to participate}

This trial has been approved by the Sichuan Regional Ethics Review Committee on Traditional Chinese Medicine (Approval number: 2019KL-026).
This trial follows the principles of the Declaration of Helsinki (Version Edinburgh 2000).

All eligible patients will be informed of the study's details and all the benefits and risks that they may take from this trial. Patients will be asked if they agree to use their data on the consent form should they choose to withdraw from the trial. Patients will also be asked for permission for the research team to share relevant data with people from the universities taking part in the research or from regulatory authorities. In addition, all researchers were trained and signed a pledge to protect the confidentiality of study participants.

\section{Consent for publication}

Study results will be published by the investigators in peer-reviewed journals or presented at conferences.

\section{Competing interests}

The authors declare that they have no competing interests.

\section{Author details}

${ }^{1}$ Acupuncture and Tuina School/The 3rd Teaching Hospital, Chengdu University of Traditional Chinese Medicine, 37\# Shierqiao Road, Chengdu 610075, Sichuan, China. ${ }^{2}$ Acupuncture-Brain Research Center, Chengdu University of Traditional Chinese Medicine, Chengdu, Sichuan, China. ${ }^{3}$ School of Administration, Chengdu University of Traditional Chinese Medicine, Chengdu, Sichuan, China. ${ }^{4}$ Department of Neurobiology and Acupuncture Research/The Third Clinical Medical College, Zhejiang Chinese Medical University, Hangzhou, Zhejiang, China. ${ }^{5}$ Key Laboratory of Sichuan Province for Acupuncture \& Chronobiology, Chengdu, Sichuan, China.

Received: 23 December 2019 Accepted: 22 October 2020

Published online: 06 November 2020

\section{References}

1. Forouzanfar MH, Moran AE, Flaxman AD, Roth G, Mensah GA, Ezzati M, et al. Assessing the global burden of ischemic heart disease, part 2: analytic methods and estimates of the global epidemiology of ischemic heart disease in 2010. Glob Heart. 2012;7:331-42 https://doi.org/10.1016/j.gheart. 2012.10.003.

2. Tarkin JM, Kaski JC. Pharmacological treatment of chronic stable angina pectoris. Clin Med (Lond). 2013;13:63-70 https://doi.org/10.7861/ clinmedicine.13-1-63.

3. Lloyd-Jones DM, Hong Y, Labarthe D, Mozaffarian D, Appel L, van Horn L, et al. Defining and setting national goals for cardiovascular health promotion and disease reduction: the American Heart Association's strategic Impact Goal through 2020 and beyond. Circulation. 2010;121:586$613 \mathrm{https}: / /$ doi.org/10.1161/CIRCULATIONAHA.109.192703.

4. Bhatt DL, Eagle KA, Ohman EM, Hirsch AT, Goto S, Mahoney EM, et al. Comparative determinants of 4-year cardiovascular event rates in stable outpatients at risk of or with atherothrombosis. JAMA. 2010;304:1350-7 https://doi.org/10.1001/jama.2010.1322.

5. Mozaffarian D, Benjamin EJ, Go AS, Arnett DK, Blaha MJ, Cushman M, et al. Heart disease and stroke statistics--2015 update: a report from the American Heart Association. Circulation. 2015;131:e29-322 https://doi.org/10.1161/CIR. 0000000000000152.

6. Ohman EM. Clinical practice. Chronic stable angina. N Engl J Med. 2016;374: 1167-76 https://doi.org/10.1056/NEJMcp1502240.

7. Brorsson B, Bernstein SJ, Brook RH, Werkö L. Quality of life of patients with chronic stable angina before and four years after coronary revascularisation compared with a normal population. Heart. 2002;87:140-5 https:/doi.org/ 10.1136/heart.87.2.140

8. Hemingway H, McCallum A, Shipley M, Manderbacka K, Martikainen P Keskimäki I. Incidence and prognostic implications of stable angina pectoris among women and men. JAMA. 2006;295:1404-11 https://doi.org/10.1001/ jama.295.12.1404

9. Montalescot G, Sechtem U, Achenbach S, Andreotti F, Arden C, Budaj A, et al. 2013 ESC guidelines on the management of stable coronary artery disease: the Task Force on the management of stable coronary artery disease of the European Society of Cardiology. Eur Heart J. 2013;34:29493003 https://doi.org/10.1093/eurheartj/eht296.

10. Xu L, Xu H, Gao W, Wang W, Zhang H, Lu DP. Treating angina pectoris by acupuncture therapy. Acupunct Electrother Res. 2013;38:17-35. 
11. Kang XZ, Xia Y. Acupuncture therapy for arrhythmia and other cardiac disorders: clinical and laboratory investigation. Zhen Ci Yan Jiu. 2009;34:41320.

12. Zhao L, Li D, Zheng H, Chang X, Cui J, Wang R, et al. Acupuncture as adjunctive therapy for chronic stable angina: a randomized clinical trial. JAMA Intern Med. 2019; https://doi.org/10.1001/jamainternmed.2019. 2407.

13. Ballegaard S, Jensen G, Pedersen F, Nissen VH. Acupuncture in severe, stable angina pectoris: a randomized trial. Acta Med Scand. 1986;220:307-13 https://doi.org/10.1111/j.0954-6820.1986.tb02770.x.

14. Meng J. The effects of acupuncture in treatment of coronary heart diseases. J Tradit Chin Med. 2004;24:16-9.

15. Liu Y, Meng HY, Khurwolah MR, Liu J-B, Tang H, Aa N, Yang Z-J. Acupuncture therapy for the treatment of stable angina pectoris: an updated meta-analysis of randomized controlled trials. Complement Ther Clin Pract. 2019;34:247-53 https://doi.org/10.1016/j.ctcp.2018.12.012.

16. Cheng S, Shao X, Liang F, Zeng F. The law of selecting acupoint treating for angina pectoris. Lishizhen Med Mater Med Res. 2014;25:913-4 [Chinese].

17. Miyazaki S, Hagihara A, Kanda R, Mukaino Y, Nobutomo K. Applicability of press needles to a double-blind trial: a randomized, double-blind, placebocontrolled trial. Clin J Pain. 2009;25:438-44 https://doi.org/10.1097/AJP. Ob013e318193a6e1.

18. Xu W, Sun D, Ye WX, Li SM, Wang FY. Therapeutic effect of intradermal needling combined with rehabilitation intervention on knee osteoarthritis in the middle and early stages. Zhongguo Zhen Jiu. 2019;39:609-12 https:// doi.org/10.13703/j.0255-2930.2019.06.011.

19. Chan AW, Tetzlaff JM, Gotzsche PC, Altman DG, Mann H, Berlin JA, et al. SPIRIT 2013 explanation and elaboration: guidance for protocols of clinical trials. BMJ. 2013;346:e7586 https://doi.org/10.1136/bmj.e7586.

20. MacPherson H, Altman DG, Hammerschlag R, Youping L, Taixiang W, White A, Moher D. Revised STandards for Reporting Interventions in Clinical Trials of Acupuncture (STRICTA): extending the CONSORT statement. PLoS Med. 2010;7:e1000261 https://doi.org/10.1371/journal.pmed.1000261.

21. Wang B, Li Y, Han Y. Guidelines for the diagnosis and treatment of stable coronary artery disease. Chin J Cardiol. 2018;46:680-94. [Chinese].

22. Sangareddi $V$, Chockalingam A, Gnanavelu G, Subramaniam T, Jagannathan $\checkmark$, Elangovan S. Canadian Cardiovascular Society classification of effort angina: an angiographic correlation. Coron Artery Dis. 2004;15:111-4.

23. Chow SC, Shao J, Wang H. Sample Size Calculations in Clinical Research, second edition. Boca Raton: Chapman and Hall/CRC Biostatistics Series; 2008.

24. Luo L, Yuan $C-K$, Yin $H-Y$, Zeng F, Tang $Y$, Yu S-G. Experience and discussion on the national standard standardized manipulation of acupuncture and moxibustion. Part 8: intradermal needle. Zhongguo Zhen Jiu. 2012;32:155-8.

25. Zhang WB, Wang YP, Li HY. Analysis on correlation between meridians and viscera in book the yellow Emperor's internal classic. Zhen Ci Yan Jiu. 2018; 43:424-9 https://doi.org/10.13702/j.1000-0607.180185.

26. Liu WT, Zhang LP, Zheng MF. Current researches and ideas regarding correlation between meridians and viscera. Zhen Ci Yan Jiu. 2018;43:430-2 https://doi.org/10.13702/j.1000-0607.180069.

27. Rong P, Zhu B, Li Y, Gao X, Ben H, Li Y, et al. Mechanism of acupuncture regulating visceral sensation and mobility. Front Med. 2011;5:151-6 https:// doi.org/10.1007/s11684-011-0129-7.

28. Fan H, Zhao L, Cui J, Wang R, Chang X, Li D, et al. Effects of acupuncture at acupoints on heart rate variability in patients with chronic stable angina pectoris. Chin J Tradit Chin Med Pharm. 2017;32:1798-803 [Chinese].

29. Ma TT, Yu SY, Li Y, Liang FR, Tian XP, Zheng H, et al. Randomised clinical trial: an assessment of acupuncture on specific meridian or specific acupoint vs. sham acupuncture for treating functional dyspepsia. Aliment Pharmacol Ther. 2012;35:552-61 https://doi.org/10.1111/j.1365-2036.2011.04979.x.

30. Yan R, Zhang Y, Lim J, Yang F, Zhou L, Lyu D, et al. The effect and biomechanical mechanisms of intradermal needle for post-stroke hemiplegia recovery: Study protocol for a randomized controlled pilot trial. Medicine (Baltimore). 2018;97:e0448 https://doi.org/10.1097/MD. 0000000000010448.

31. Bae DB, Lyu YS, Park JM, Kang HW, Lyu YS. A preliminary comparison of efficacy of needle-embedding therapy with acupuncture for chronic tension-type headache patients. J Orient Neuropsychiatry. 2013;24:45-62 https://doi.org/10.7231/jon.2013.24.1.045.

32. Kim YS, Lee SH, Jung WS, Park SU, Moon SK, Ko CN, et al. Intradermal acupuncture on shen-men and nei-kuan acupoints in patients with insomnia after stroke. Am J Chin Med. 2004;32:771-8 https://doi.org/10. 1142/S0192415X04002399.

33. Deng J, Fei LB, Zhou JH, Wu S, Huang W, Chen X. Needle- embedding therapy combined with basic treatment for stable angina pectoris. World J Acupunct Moxibustion. 2018;28:81-85+151 https://doi.org/10.1016/j.wjam. 2018.05.004.

34. Fraker TD, JR FSD, Gibbons RJ, Abrams J, Chatterjee K, Daley J, et al. 2007 chronic angina focused update of the ACC/AHA 2002 guidelines for the management of patients with chronic stable angina: a report of the American College of Cardiology/American Heart Association Task Force on Practice Guidelines Writing Group to develop the focused update of the 2002 guidelines for the management of patients with chronic stable angina. J Am Coll Cardiol. 2007;50:2264-74 https://doi.org/10.1016/j.jacc.2007.08.002.

\section{Publisher's Note}

Springer Nature remains neutral with regard to jurisdictional claims in published maps and institutional affiliations.
Ready to submit your research? Choose BMC and benefit from:

- fast, convenient online submission

- thorough peer review by experienced researchers in your field

- rapid publication on acceptance

- support for research data, including large and complex data types

- gold Open Access which fosters wider collaboration and increased citations

- maximum visibility for your research: over $100 \mathrm{M}$ website views per year

At BMC, research is always in progress.

Learn more biomedcentral.com/submissions 